\title{
Perceived quality and availability of fruit and vegetables are associated with perceptions of fruit and vegetable affordability among socio-economically disadvantaged women
}

\author{
Lauren K Williams*, Lukar Thornton, David Crawford and Kylie Ball \\ Centre for Physical Activity and Nutrition Research, School of Exercise and Nutrition Sciences, Deakin University, \\ 221 Burwood Highway, Burwood, Victoria 3125, Australia
}

Submitted 14 March 2011: Accepted 17 November 2011: First published online 10 January 2012

\begin{abstract}
Objective: Perceptions that fruit and vegetables are expensive have been found to be associated with lower consumption of fruit and vegetables among disadvantaged women; however, the determinants of these perceptions are relatively unknown. The purpose of the current paper is to examine whether perceived availability and quality of fruit and vegetables, and social support for healthy eating, are associated with perceptions of fruit and vegetable affordability among women residing in disadvantaged neighbourhoods.

Design: Cross-sectional self-report survey.

Setting: The study was conducted in Melbourne, Australia.

Subjects: An Australian sample of 4131 women, aged 18-45 years, residing in neighbourhoods ranked in the lowest Victorian tertile of relative disadvantage by the Australian Bureau of Statistics, an index that considers aspects of disadvantage such as residents' income, education, motor vehicle access and employment.

Results: Results showed that irrespective of education, income and other key covariates, women who perceived poor availability and quality of fruit and vegetables in their local neighbourhood were more likely to perceive fruit and vegetables as expensive.

Conclusions: Our results suggest that perceptions of fruit and vegetable affordability are not driven exclusively by lack of financial or knowledge-related resources, but also by women's psychological response and interpretation of their local nutrition environment.
\end{abstract}

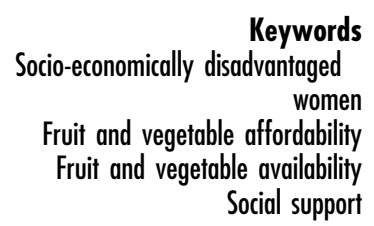

Socio-economic gradients in dietary intakes among women are well established: compared with those of high socio-economic position (SEP), women of low SEP (e.g. women with low education, low income and/or residing in disadvantaged neighbourhoods) consume diets that are less consistent with guidelines for health, including lower consumption of fruit and vegetables ${ }^{(1-3)}$. One explanation for this socio-economic gradient in fruit and vegetable consumption is that women of low SEP are more likely than their more advantaged counterparts to perceive fruit and vegetables as expensive ${ }^{(4-10)}$. Women's perceptions of fruit and vegetable affordability, rather than actual prices of fruit and vegetables, have been found to significantly mediate the relationship between SEP and fruit and vegetable consumption ${ }^{(11,12)}$. Little is known, however, about what determines negative costrelated perceptions of fruit and vegetables.

There are individual- and area-level explanations that may account for negative perceptions about the affordability of fruit and vegetables held by low-SEP women.
Plausibly, negative perceptions of fruit and vegetable affordability result from real financial pressure that reduces capacity to purchase healthy food or the absence of nutrition education that impacts knowledge of what varieties of fruit and vegetables are affordable. Negative perceptions about fruit and vegetable affordability experienced by low-SEP women may also be attributable to high actual costs of locally available fruit and vegetables and the high cost of fruit and vegetables relative to other foods. However, in contrast to results found predominantly in US studies ${ }^{(13)}$, there is limited evidence that in Australia the cost of fruit and vegetables is higher for those residing in socio-economically disadvantaged neighbourhoods ${ }^{(14-16)}$. Further, although some healthy food options are more expensive than less healthy options, there is limited evidence that these actual or objective costs of healthy food (e.g. fruit and vegetables, low-fat dairy, lean meat and non-white bread) are related to perceptions of healthy food affordability ${ }^{(17,18)}$. Together such findings suggest that perceptions of fruit and vegetable affordability 
may be influenced more by non-economic considerations, rather than actual price alone.

In addition to fruit and vegetable affordability, other environmental perceptions that have been found to be associated with poorer consumption of fruit and vegetables include lower perceived availability and quality of healthy food options ${ }^{(4,10,19)}$ and also lower perceived social support from family and friends to consume a healthy diet ${ }^{(1,4,5,7,20)}$. It is possible that women who perceive poor quality and availability of fruit and vegetables consider fruit and vegetables to be expensive as they do not represent good 'value for money' or are too difficult to obtain. For instance, stable or consistent prices for fruit and vegetables may be considered expensive if the produce is spoiled (bruised, wilted, etc.) and variety is limited (e.g. varieties consumers know to be cheaper are not available). Likewise, those with low social support for healthy eating may perceive the cost of fruit and vegetables as higher than those who have support, because the effort required to persuade family members to eat these foods may represent a perceived 'cost' to increased fruit and vegetable purchase and consumption.

Given that perceptions of fruit and vegetable affordability appear to play an important role in shaping women's diets, particularly among low-SEP women, it is important to understand factors that influence these perceptions in order to determine the best avenue for intervention. To our knowledge an assessment of determinants of perceived affordability of fruit and vegetables, other than actual cost, has not been conducted. The aim of the current paper is to assess whether perceived availability and quality of fruit and vegetables, and social support for healthy eating, are associated with negative perceptions of fruit and vegetable affordability among women residing in disadvantaged neighbourhoods.

\section{Methods}

\section{Sample}

Analyses presented are based on data from a sample of 4131 women who participated in the Resilience for Eating and Physical Activity Despite Inequality (READI) study, a longitudinal cohort study examining resilience to obesity among women and children residing in socially and economically disadvantaged rural and urban areas of Victoria, Australia ${ }^{(21-23)}$. Areas (defined by the administrative unit of suburb) considered disadvantaged were those ranked in the lowest Victorian tertile of disadvantage using the Socio-Economic Index for Areas (SEIFA) Index of Relative Socio-economic Disadvantage (IRSD). The SEIFA IRSD is an aggregate index created from Australian census data that considers residents' level of income, education, car ownership and employment. Forty urban and forty rural disadvantaged neighbourhoods were randomly selected for the READI study. The forty urban areas were sampled from within metropolitan Melbourne or a $10 \mathrm{~km}$ radius of the centroid of rural cities (defined by the Regional Infrastructure Development Fund Act, 1999). The forty rural areas were sampled from areas falling outside the urban areas with a population of $\geq 1200$ and within $200 \mathrm{~km}$ of Melbourne. For the READI study, all women completed a baseline survey that assessed individual, social and environmental factors potentially associated with physical activity, diet and weight.

\section{Procedure}

The study was approved by the Deakin University Human Research Ethics Committee. Within each of the eighty disadvantaged rural and urban areas selected, 150 women aged 18-45 years were randomly selected from the Australian electoral roll (the electoral role is compulsory for Australian citizens). In areas with less than 150 eligible women, all eligible women were sampled. Between August 2007 and January 2008, 11940 women were selected and mailed an invitation, a survey, a consent form and a \$AU 1 lottery ticket as a small compensation for their time. A reminder was sent to non-respondents at $10 \mathrm{~d}$ and again at $20 \mathrm{~d}$ after the initial survey pack was mailed. Of 11940 women selected, 4934 women completed a survey with the response rate being slightly higher among rural than urban women (39\%v. 34\%). Although this response rate is quite low, it is not unexpected given the focus on women residing in disadvantaged neighbourhoods. Data were excluded for women who had moved from the sampled suburb before survey completion ( $n$ 571), who had completed the survey but were not the intended participants ( $n$ 3), who withdrew their data after completing the survey ( $n 2)$, who were aged under 17 years or over 46 years ( $n 9$ ), or who had missing data on any of the variables considered herein ( $n$ 218).

\section{Measures}

The measures used in the READI study were based on social ecological theories and theories explaining socioeconomic variations in diet ${ }^{(4,24)}$. Key elements of the hypothesized model include intrapersonal variables and social and physical environmental variables. The perceived fruit and vegetable affordability scale and the perceived availability and quality of fruit and vegetables scales were developed specifically for use in the READI study, on the basis of previous research and qualitative data on determinants of diet in low-SEP women ${ }^{(5)}$. The social support for healthy eating scales were adapted from a well-validated scale ${ }^{(25)}$. These measures are described in more detail below.

\section{Outcome measure: perceived fruit and vegetable affordability}

Participants were asked to two separate questions: 'I do not buy many fruit because they cost too much' and 'I do not buy many vegetables because they cost too much'. 
The five response categories were: strongly disagree (1); disagree (2); neither agree nor disagree (3); agree (4); and strongly agree (5). Responses were retained as an ordinal scale.

\section{Independent measures: perceived availability and quality of fruit and vegetables and perceived social support for bealthy eating}

Four independent measures were included in the current paper: (i) perceived availability of fruit and vegetables; (ii) perceived quality of fruit and vegetables; (iii) perceived social support from family to eat healthy food; and (iv) perceived social support from friends/work colleagues to eat healthy foods. To assess perceived availability of fruit and vegetables, participants were asked: 'A large selection of fruit and vegetables are available in my neighbourhood'. To assess perceived quality of fruit and vegetables, participants were asked: 'The fresh fruit and vegetables in my neighbourhood are of high quality'. For both availability and quality questions, the five response categories were: strongly disagree (1); disagree (2); neither agree nor disagree (3); agree (4); and strongly agree (5). Responses were dichotomised into 'do not agree' ( $\leq 3)$ and 'agree' ( $\geq 4)$.

To assess social support from family members for healthy eating, participants were asked three questions: 'During the past year, how often did members of your family (including spouse/partner)...' (i) '...eat healthy low-fat foods with you?' (ii) '....encourage you to eat healthy low-fat foods?' and (iii) '... discourage you from eating unhealthy foods?' The five response categories were: never (1); rarely (2); a few times (3); often (4); and very often (5). Responses for these three items were summed to create a Family Social Support scale that had a range of 3 (i.e. answered 'never' to all three items) to 15 (i.e. answered 'very often' to all three items), with higher scores representing more social support. Cronbach's $\alpha$ (a measure of internal consistency reliability) for the Family Social Support scale was $0 \cdot 80$. The Friends' and Work Colleagues' Social Support scale employed the same methodology and items as those used to develop the Family Social Support scale with the preamble to the three questions stating: 'During the past year, how often did friends or work colleagues...'. Cronbach's $\alpha$ for the Friends' and Work Colleagues' Social Support scale was $0 \cdot 75$.

\section{Covariates: selected participant demographics}

Covariates included were: education, household income, age, number of children, rurality and country of birth. Women self-reported their highest level of education achieved (classified as low (no formal qualifications/Year 10 or equivalent), medium (Year 12 or equivalent/trade/ apprenticeship/certificate or diploma) or high (university undergraduate or postgraduate degree)), average annual household income including wages, salary, pensions and allowances (classified as low (\$AU 0-36 999), medium
(\$AU 37 000-77999), high ( $\geq$ \$AU 78000 ) and undisclosed), age (in years), number of children (dichotomised as 'none' and 'one or more' due to low responses for the upper response options), rurality (classified as urban, inner rural and outer rural), clustering of suburbs and country of birth (dichotomised as 'Australian' or 'other').

\section{Statistical analyses}

Analyses were conducted using the statistical software packages SPSS version $17 \cdot 0$ (SPSS Inc., Chicago, IL, USA) and STATA/SE version $10 \cdot 1$ (StataCorp LP, College Station, TX, USA). Exploratory factor analysis with direct oblimin rotation and zero-order correlations were conducted to confirm independence between the variables measured. Multinomial logistic regression was used to assess the bivariate and multivariate associations between all four independent variables and (i) perceived fruit affordability and (ii) perceived vegetable affordability. Analyses were conducted without (unadjusted) and with (adjusted) covariates and adjusted for clustering of suburbs. In the final models, only those found to have a significant bivariate relationship with perceived fruit and vegetable affordability were included in the multivariate models. A $P$ value of $<0.05$ was considered as statistically significant.

\section{Results}

The mean age of the women was 34 years, and the majority of them had children $(60.9 \%)$, were born in Australia (89.4\%) and resided in rural areas (63.5\%). The rates of low, medium and high education level were $21 \cdot 9 \%, 51 \cdot 8 \%$ and $26 \cdot 3 \%$, respectively, while the rates of household income for low, medium, high and undisclosed were $25 \cdot 5 \%, 35 \cdot 5 \%, 17 \cdot 9 \%$ and $20.9 \%$, respectively. In the bivariate analyses (data not shown) women who perceived poor availability and quality of fruit and vegetables and less social support from family members to eat healthily were more likely to perceive fruit and vegetables to be expensive. There was no significant relationship between social support from friends/work colleagues to eat healthily and perceptions of fruit or vegetable affordability, and as such this variable was not entered into the multivariate analyses.

Table 1 shows that after adjusting for key covariates, compared with women who perceived a good selection of fruit and vegetables available in their local neighbourhood, women who perceived there to be a poor selection of fruit and vegetables available in their local neighbourhood were more likely to agree that fruit and vegetables are expensive. Compared with women who perceived the quality of fruit and vegetables in their neighbourhood to be good, women who perceived the quality to be poor were more than three times more likely to agree that fruit and vegetables are expensive. Compared with women who reported low support from 
Table 1 Adjusted odds ratios and $95 \%$ confidence intervals for the likelihood of agreeing that fruit and vegetables cost too much according to perceived fruit and vegetable availability and quality and social support from family for healthy eating: sample of socio-economically disadvantaged women, aged 18-45 years, Victoria, Australia

\begin{tabular}{|c|c|c|c|c|c|c|c|c|}
\hline \multirow[b]{3}{*}{ Independent variable } & \multicolumn{8}{|c|}{$\begin{array}{c}\text { I do not buy many fruit because they cost too much }(n 4131) \\
\text { Reference category: Strongly disagree }(n 892)\end{array}$} \\
\hline & \multicolumn{2}{|c|}{ Disagree $(n$ 1830) } & \multicolumn{2}{|c|}{$\begin{array}{l}\text { Neither agree nor } \\
\text { disagree }(n 728)\end{array}$} & \multicolumn{2}{|c|}{ Agree $(n 555)$} & \multicolumn{2}{|c|}{ Strongly agree $(n 126)$} \\
\hline & OR & $95 \% \mathrm{Cl}$ & OR & $95 \% \mathrm{Cl}$ & OR & $95 \% \mathrm{Cl}$ & OR & $95 \% \mathrm{Cl}$ \\
\hline \multicolumn{9}{|l|}{ Model $1 \dagger$} \\
\hline \multicolumn{9}{|l|}{ Availability } \\
\hline Perceive good selection of fruit and vegetables available in neighbourhood $(n 941)$ & $1 \cdot 00$ & & $1 \cdot 00$ & & $1 \cdot 00$ & & $1 \cdot 00$ & \\
\hline Perceive poor selection of fruit and vegetables available in neighbourhood ( $n$ 3190) & $0 \cdot 86$ & $0.69,1.06$ & $1 \cdot 14$ & $0 \cdot 85,1.53$ & $1 \cdot 48^{\star \star}$ & $1 \cdot 12,1 \cdot 97$ & $1 \cdot 33$ & $0 \cdot 84,2 \cdot 11$ \\
\hline \multicolumn{9}{|l|}{ Quality } \\
\hline Perceived high quality of fruit and vegetables in neighbourhood $(n 1765)$ & $1 \cdot 00$ & & $1 \cdot 00$ & & $1 \cdot 00$ & & $1 \cdot 00$ & \\
\hline Perceived low quality of fruit and vegetables in neighbourhood ( $n 2366)$ & $1 \cdot 63^{\star \star}$ & $1 \cdot 35,1 \cdot 98$ & $2 \cdot 81^{\star \star}$ & $2 \cdot 22,3 \cdot 57$ & $3 \cdot 24^{\star \star}$ & $2 \cdot 49,4 \cdot 22$ & $2 \cdot 75^{\star \star}$ & $1 \cdot 75,4 \cdot 32$ \\
\hline \multicolumn{9}{|l|}{ Family social support } \\
\hline \multirow[t]{4}{*}{ Support from family members to eat healthy foods } & 0.99 & $0 \cdot 96,1 \cdot 02$ & $0 \cdot 96^{*}$ & $0.93,0.99$ & 0.97 & $0.93,1.00$ & 0.96 & $0.90,1.02$ \\
\hline & \multicolumn{8}{|c|}{$\begin{array}{c}\text { I do not buy many vegetables because they cost too much ( } n \text { 4131) } \\
\text { Reference category: Strongly disagree }(n \text { 1038) }\end{array}$} \\
\hline & \multicolumn{2}{|c|}{ Disagree ( $n$ 2032) } & \multicolumn{2}{|c|}{$\begin{array}{l}\text { Neither agree nor } \\
\text { disagree }(n 674)\end{array}$} & \multicolumn{2}{|c|}{ Agree $(n 315)$} & \multicolumn{2}{|c|}{ Strongly agree $(n 72)$} \\
\hline & OR & $95 \% \mathrm{Cl}$ & OR & $95 \% \mathrm{Cl}$ & OR & $95 \% \mathrm{Cl}$ & OR & $95 \% \mathrm{Cl}$ \\
\hline \multicolumn{9}{|l|}{$\begin{array}{l}\text { Model } 2+ \\
\text { Availability }\end{array}$} \\
\hline Perceive good selection of fruit and vegetables available in neighbourhood ( $n$ 941) & $1 \cdot 00$ & & $1 \cdot 00$ & & $1 \cdot 00$ & & $1 \cdot 00$ & \\
\hline Perceive poor selection of fruit and vegetables available in neighbourhood ( $n 3190)$ & 0.92 & $0.73,1 \cdot 15$ & $1 \cdot 34^{*}$ & $1 \cdot 03,1 \cdot 75$ & $1 \cdot 60^{* *}$ & $1 \cdot 16,2 \cdot 22$ & $1 \cdot 41$ & $0 \cdot 78,2 \cdot 54$ \\
\hline \multicolumn{9}{|l|}{ Quality } \\
\hline Perceived high quality of fruit and vegetables in neighbourhood ( $n$ 1765) & $1 \cdot 00$ & & $1 \cdot 00$ & & $1 \cdot 00$ & & $1 \cdot 00$ & \\
\hline Perceived low quality of fruit and vegetables in neighbourhood ( $n$ 2366) & $1 \cdot 67^{\star \star}$ & $1 \cdot 40,1 \cdot 98$ & $2 \cdot 93^{\star \star}$ & $2 \cdot 31,3 \cdot 73$ & $3 \cdot 22^{\star *}$ & $2 \cdot 46,4 \cdot 19$ & $2 \cdot 85^{\star \star}$ & $1 \cdot 63,4.96$ \\
\hline \multicolumn{9}{|l|}{ Family social support } \\
\hline Support from family members to eat healthy foods & 0.99 & $0.97,1.02$ & $0.96^{*}$ & $0.93,0.99$ & 0.97 & $0.93,1.02$ & 0.98 & $0.91,1.06$ \\
\hline
\end{tabular}

tModel adjusted for education, household income, age, number of children, rurality, clustering of suburbs and country of birth 
family members to eat healthily, women who reported receiving support from family members to eat healthily were less likely to neither agree nor disagree that fruit and vegetables are expensive.

\section{Discussion}

The current study assessed whether perceived availability and quality of fruit and vegetables, and social support for healthy eating, were associated with negative perceptions of fruit and vegetable affordability among women residing in disadvantaged neighbourhoods. To our knowledge, the current study is the first to assess determinants, other than actual cost, of perceived affordability of fruit and vegetables. Our findings suggest that irrespective of education, income and other key covariates, women residing in disadvantaged neighbourhoods who reported poor availability and quality of fruit and vegetables in their local area were more likely to perceive fruit and vegetables as expensive.

Potential mechanisms to explain the relationship between perceptions of availability and quality of fruit and vegetables and perceptions of fruit and vegetable affordability include the possibility that women who perceive fruit and vegetables to be expensive maintain more negative perceptions of the food environment generally. It is also possible that women who perceive the quality of fruit and vegetables to be poor perceive prices to be high as they represent poor value for money (e.g. price of fruit may be considered high for produce that is not in season, not fresh or nutritious and has a short shelf-life). Our results highlight that perceptions of quality may also influence attitudes towards affordability independent of actual income and as such provide a potentially modifiable strategy to consider when price-reduction or cost-related approaches to nutrition interventions are employed.

Further, it is possible that women who perceive poor availability of fruit and vegetables may perceive the cost of fruit and vegetables to be high if they believe that limited availability drives higher prices and leads to cost comparisons of other less healthy food options. Previous qualitative research has shown that price considerations of healthy food are linked to perceptions of availability of seasonal produce (that is cheaper) and perceived availability of cheaper less healthy options ${ }^{(5)}$. Given that perceptions of availability of healthy produce are related to consumption of healthy food among low-SEP women ${ }^{(8)}$ and our results show that perceptions of availability are also related to perceptions of affordability of healthy food, it may be useful to include tailored information about availability of healthy produce in nutrition promotion interventions among low-SEP women.

Our results also indicated that social support from family, friends and work colleagues was generally not associated with perceptions of fruit and vegetable affordability after taking key covariates into account. It is possible that for disadvantaged women, who may have limited financial resources, social influences are less relevant to cost-related perceptions than those attached to attributes such as 'value for money' and availability. Alternatively, the lack of a significant association could reflect a mismatch between outcomes assessed by these independent and dependent variables (i.e. use of the term 'healthy foods' for the social support variables rather than 'fruit and vegetables' that was used for the outcome variable).

Some limitations of the current study are worth noting. As is common in survey studies among low-SEP women, the response rate for participation was lower than that observed in studies of the general population and as such participants may be those who are more interested and more adherent to nutrition-related guidelines. Further limitations include the cross-sectional design and reliance on self-report data and subjective measures. It is possible that other 'non-financial' costs, not measured in the current study, are associated with perceptions of fruit and vegetable affordability. For instance, the time cost related to preparing fruit and vegetables ${ }^{(5)}$ and increased wastage of fruit and vegetables (if cooked and disliked by family members, or due to short shelf-life ${ }^{(26,27)}$ are important barriers to fruit and vegetable consumption among lowSEP women, and plausibly interconnected with perceptions around cost. As we did not assess cost, availability and quality of fruit and vegetables objectively we cannot rule out that the associations among perceptions observed here reflect associations among objective measures of these constructs. Further research is needed to explore the relationships between a range of objective and subjective food environment measures and affordability of fruit and vegetables. This will enable researchers to map the pathway and interactions between environmental cognitions, the environment and nutrition-related behaviour.

\section{Conclusions}

Cost has been consistently cited as one of the most common barriers to fruit and vegetable consumption ${ }^{(9,10)}$ and remains an important consideration in nutrition promotion, particularly among disadvantaged women where resources are often limited. The purpose of the present investigation was to answer why low-SEP women perceive fruit and vegetables to be expensive. The answer is that the broader perceptions of the local nutrition environment experienced by low-SEP women are important and potentially modifiable determinants of women's perceptions of healthy food affordability. These results are independent of household income and personal education and suggest that perceptions of fruit and vegetable affordability are not driven exclusively by lack of financial or knowledge-related resources. Improving nutrition among low-SEP women by targeting issues surrounding the cost of healthy food requires consideration 
not only of individual resources, but also women's psychological response and interpretation of their local nutrition environment. The results from the current study offer important insights into understanding potential cognitive mechanisms associated with perceptions of food affordability and may prove fruitful in assisting nutrition interventions that target cost as a barrier to consumption among disadvantaged women.

\section{Acknowledgements}

This work was supported by the National Health and Medical Research Council (ID 374241). L.K.W. and L.T. are supported by a National Health Medical Research Council Capacity Building Grant (ID 425845). K.B. is funded by a National Health Medical Research Council Senior Research Fellowship (ID 479513) and D.C. is funded by a VicHealth Fellowship. The authors declare there are no conflicts of interest. L.K.W. wrote the manuscript; L.T., K.B. and D.C. assisted with writing the manuscript; L.K.W. and L.T. analysed the data; K.B. and D.C. designed the READI study. The authors acknowledge the support and guidance from Dr Clare Hume and Dr Michelle Jackson who contributed to the project's inception, coordination and execution, and to Gavin Abbott for his statistical support.

\section{References}

1. Ball K, Crawford D \& Mishra G (2006) Socio-economic inequalities in women's fruit and vegetable intakes: a multilevel study of individual, social and environmental mediators. Public Health Nutr 9, 623-630.

2. Irala-Estévez JD, Groth M, Johansson L et al. (2000) A systematic review of socio-economic differences in food habits in Europe: consumption of fruit and vegetables. Eur J Clin Nutr 54, 706-714.

3. Beydoun MA \& Wang Y (2008) How do socio-economic status, perceived economic barriers and nutritional benefits affect quality of dietary intake among US adults? Eur J Clin Nutr 62, 303-313.

4. Kamphuis CB, van Lenthe FJ, Giskes K et al. (2007) Perceived environmental determinants of physical activity and fruit and vegetable consumption among high and low socioeconomic groups in the Netherlands. Health Place 13, 493-503.

5. Inglis V, Ball K \& Crawford D (2005) Why do women of low socioeconomic status have poorer dietary behaviours than women of higher socioeconomic status? A qualitative exploration. Appetite 45, 334-343.

6. Turrell G \& Kavanagh AM (2006) Socio-economic pathways to diet: modelling the association between socio-economic position and food purchasing behaviour. Public Health Nutr 9, 375-383.

7. Yeh MC, Ickes SB, Lowenstein LM et al. (2008) Understanding barriers and facilitators of fruit and vegetable consumption among a diverse multi-ethnic population in the USA. Health Promot Int 23, 42-51.

8. Williams L, Ball K \& Crawford D (2010) Why do some socioeconomically disadvantaged women eat better than others? An investigation of the personal, social and environmental correlates of fruit and vegetable consumption. Appetite 55, 441-446.
9. Eikenberry N \& Smith C (2004) Healthful eating: perceptions, motivations, barriers, and promoters in low-income Minnesota communities. J Am Diet Assoc 104, 1158-1161.

10. Pollard J, Kirk SF \& Cade JE (2002) Factors affecting food choice in relation to fruit and vegetable intake: a review. Nutr Res Rev 15, 373-387.

11. Inglis V, Ball K \& Crawford D (2008) Socioeconomic variations in women's diets: what is the role of perceptions of the local food environment? J Epidemiol Community Health 62, 191-197.

12. Thornton LE, Crawford DA \& Ball K (2010) Neighbourhoodsocioeconomic variation in women's diet: the role of nutrition environments. Eur J Clin Nutr 64, 1423-1432.

13. Cummins S \& Macintyre S (2006) Food environments and obesity - neighbourhood or nation? Int J Epidemiol 35, 100-104.

14. Winkler E, Turrell G \& Patterson C (2006) Does living in a disadvantaged area entail limited opportunities to purchase fresh fruit and vegetables in terms of price, availability, and variety? Findings from the Brisbane Food Study. Health Place 12, 741-748.

15. Ball K, Timperio A \& Crawford D (2009) Neighbourhood socioeconomic inequalities in food access and affordability. Health Place 15, 578-585.

16. Pearce J, Witten K, Hiscock R et al. (2007) Are socially disadvantaged neighbourhoods deprived of health-related community resources? Int J Epidemiol 36, 348-355.

17. Giskes K, Van Lenthe FJ, Brug J et al. (2007) Socioeconomic inequalities in food purchasing: the contribution of respondent-perceived and actual (objectively measured) price and availability of foods. Prev Med 45, 41-48.

18. Williams LK, Thornton L, Ball K et al. (2011) Is the objective food environment associated with perceptions of the food environment? Public Health Nutr (Epublication ahead of print version).

19. Raine KD (2005) Determinants of healthy eating in Canada: an overview and synthesis. Can J Public Health 96, Suppl. 3, S8-S14.

20. Anderson ES, Winett RA \& Wojcik JR (2007) Self-regulation, self-efficacy, outcome expectations, and social support: social cognitive theory and nutrition behavior. Ann Behav Med 34, 304-312.

21. MacFarlane A, Abbott G, Crawford D et al. (2010) Personal, social and environmental correlates of healthy weight status amongst mothers from socioeconomically disadvantaged neighborhoods: findings from the READI study. Int $J$ Behav Nutr Phys Act 7, 23.

22. Cleland V, Hume C, Crawford D et al. (2010) Urban-rural comparison of weight status among women and children living in socioeconomically disadvantaged neighbourhoods. Med J Aust 192, 137-140.

23. Cleland V, Ball K, Hume C et al. (2010) Individual, social and environmental correlates of physical activity among women living in socioeconomically disadvantaged neighbourhoods. Soc Sci Med 70, 2011-2018.

24. Ball K, Abbott G, Cleland V et al. (2011) Resilience to obesity among socioeconomically disadvantaged women: the READI study. Int J Obes (Lond) (Epublication ahead of print version).

25. Sallis J, Grossman R, Pinski R et al. (1987) The development of scales to measure social support for diet and exercise behaviors. Prev Med 16, 825-836.

26. John JH \& Ziebland S (2004) Reported barriers to eating more fruit and vegetables before and after participation in a randomized controlled trial: a qualitative study. Health Educ Res 19, 165-174.

27. Rose D (2007) Food Stamps, the Thrifty Food Plan, and meal preparation: the importance of the time dimension for US nutrition policy. J Nutr Educ Behav 39, 226-232. 